\section{P63 (continued)}

Study Design, Setting, Participants: The authors used case study methods in 19 urban public schools: 120 interviews with program and school staff, 7 student focus groups, and 91 school observations.

Measurable Outcome/Analysis: We coded data in Dedoose and met regularly to discuss emerging themes. Codes included the taxonomy of implementation outcomes (Proctor et al, 2010). This analysis draws from codes that focus on what program participants and staff see as the most successful and valuable parts of the program. We mapped our findings onto the Theory of Planned Behavior (Azjen, 1991), which posits that before behavior can change, 3 factors must shift (attitudes, perceived behavioral control, and subjective norms).

Results: Participants indicated that the most important aspects of the program are "exposure" to new foods (attitude change), access to healthy foods (shift in perceived control), and school culture around health (change in subjective norms).

Conclusions: We propose creating a new conceptual model for evaluating SNAP-Ed programming in a school, particularly for Policy, Systems, and Environmental (PSE) work, that aligns outcomes to participants' own understandings of program impacts. This model would capture changes in attitudes, perceived behavioral control, and subjective norms earlier in program implementation. These are vital to sustained behavior change but often overlooked when measuring nutrition interventions.

Funding: Supplemental Nutrition Assistance Program Education.

\section{P64 Fruit and Vegetable Prescription Program Improves Dietary Intake and Health Beliefs of At-Risk Pediatrics and Caregivers}

Siew Guan Lee, MS, RDN, siewguanl@uidaho.edu, University of Idaho Extension, 630 Addison Ave W, Suite 1600, Twin Falls, ID, 83301; Annie Roe, PhD, RDN, University of Idaho Extension; Cammie Jayo, BS, University of Idaho Extension

Objective: Examine the effectiveness of a Fruit and Vegetable Prescription (FV Rx) program for at-risk children and caregivers.

Use of Theory or Research: Higher fruit and vegetable intakes have been associated with reduced risk of chronic disease. Growing evidence supports the use of FV Rx programs to improve fruit and vegetable consumption among participants. Additional research is needed to determine effects of such programs on pediatric patients and caregivers.

Target Audience: Pediatric patients deemed to be at risk of diet-related chronic disease, and their caregivers.

Program Description: Participants screened by clinicians were prescribed fruits and vegetables for 6 months (October 2018-March 2019). Caregivers received a FV Rx card (\$60 per month) to purchase produce at participating stores and were offered nutrition education and grocery shopping classes.
Evaluation Methods: Surveys were administered to collect demographic data; pre/post caregiver and child dietary intake; pre/post attitudes, beliefs, and health status (Likert scale); and overall experience participating in the program. Percent of participants reporting increased dietary intake or a positive shift on the Likert scale from preto post-program was recorded.

Results: Ninety-four dyads completed pre/post surveys (children: age $8.9 \pm 5.0,50 \%$ males, $78.5 \%$ Hispanic). Most families (60\%) were currently receiving or had received SNAP benefits in the past and used FV Rx cards once or twice a month (57\%). Caregivers reported participating stores were easy to get to (88\%), and they felt comfortable using the card (69\%). Caregivers reported positive shifts in children's dietary intake including fruits $(62 \%)$, green leafy salad (56\%), and beans (45\%). More adults met the dietary guidelines for fruit (59\%) and vegetable intake (25\%) post-program vs pre-program (37\% fruit, $6 \%$ vegetable). Caregivers (42-57\%) also reported positive shifts in perceived personal and child health and diet status.

Conclusions: By increasing access and affordability of healthy foods through a FV Rx program in clinical settings, children and their caregivers can make positive shifts to improve dietary intake and health beliefs which may reduce risk of chronic disease.

Funding: Supplemental Nutrition Assistance Program Education, Chobani.

\section{P65 Navigating Food Insecurity at College: Examining Student Experiences and Perspectives on Solutions}

Tracey Thomas, DPH, CHES, thoma3tl@jmu.edu, James Madison University, MSC 4301235 Martin Luther King Jr Way, Harrisonburg, VA, 22807; Jamie Williams, M.Ed., James Madison University; Lilliokanaio Peaslee, PhD, James Madison University; Livvy Call, BS, James Madison University; Jeremy Hawkins, BA, James Madison University

Background: There is growing awareness of food insecurity among college students, with several studies documenting its presence and recommendations for addressing it. The majority of studies are quantitative and fail to offer insight into how students view food insecurity and what they and their universities can do about it. This study provides that perspective.

Objective: To examine how students experiencing food insecurity make sense of, navigate, and recommend addressing it.

Study Design, Setting, Participants: An anonymous survey was administered at a Mid-Atlantic university using Qualtrics and elicited 1,283 responses (93.1\% undergraduate; $6.9 \%$ graduate). Following the survey, three 90-minute focus groups were conducted with 13 students $(84.6 \%$ undergraduate; $15.4 \%$ graduate) who self-identified as food insecure.

Measurable Outcome/Analysis: The 68-item survey included questions regarding students' background, finances, and food security status (using the 6-item USDA-FSS Short Form survey). The 19-item focus group guide 